\title{
Estrogen weakens muscle endurance via estrogen receptor-p38 MAPK-mediated orosomucoid (ORM) suppression
}

\author{
Yang Sun ${ }^{1,3}$, Zhen Qin ${ }^{1,3}$, Jing-Jing Wan ${ }^{1}$, Peng-Yuan Wang ${ }^{1}$, Yi-Li Yang ${ }^{2}$, Jian-Guang Yu ${ }^{1}$, Bo-Han $\mathrm{Hu}^{1}$, \\ Ding-Feng Su${ }^{1}$, Zhu-Min $\mathrm{Luo}^{1}$ and Xia Liu ${ }^{1}$
}

Gender differences in fatigue manifest as females being more prone to feel exhaustion and having lower muscle endurance. However, the mechanisms of these effects remain unclear. We investigated whether orosomucoid, an endogenous anti-fatigue protein that enhances muscle endurance, is involved in this regulation. Female rats exhibited lower muscle endurance, and this gender difference disappeared in orosomucoid-1-deficient mice. Female rats also exhibited weaker orosomucoid induction in serum, liver and muscle in response to fatigue compared with male rats. Ovariectomy elevated orosomucoid levels and increased swimming time, and estrogen replenishment reversed these effects. Exogenous estrogen treatment in male and female mice produced opposite effects. Estrogen decreased orosomucoid expression and its promoter activity in $\mathrm{C} 2 \mathrm{C} 12$ muscle and Chang liver cells in vitro, and estrogen receptor or p38 mitogen-activated protein kinase blockade abolished this effect. Therefore, estrogen negatively regulates orosomucoid expression that is responsible for the weaker muscle endurance in females. Experimental \& Molecular Medicine (2018) 50, e463; doi:10.1038/emm.2017.307; published online 30 March 2018

\section{INTRODUCTION}

Fatigue generally manifests as a reversible decline in performance by affected individuals, ${ }^{1}$ and it is divided into central fatigue and physical fatigue. Patients with central fatigue, especially chronic fatigue syndrome, always complain about reduced physical endurance, ${ }^{2,3}$ and physical fatigue is generally accompanied by deceased muscle function and fuel depletion. Muscle fatigue is a common phenomenon that is defined as an exercise-induced reduction in the ability of a muscle or muscle group to generate maximal force ${ }^{4}$ that limits daily life, athletic performance or other strenuous or prolonged activity. Muscle endurance is usually used to evaluate muscle resistance to fatigue that refers to the capacity of a muscle or a group of muscles to sustain repeated contractions against a resistance (for example, a given load or repetitive stimuli) for an extended period of time. Fatigue index is used to measure muscle endurance. ${ }^{5,6}$

Gender difference in fatigue and muscle endurance is an important factor in clinical practice. ${ }^{7,8}$ Females are more prone to complain of fatigue and require greater recovery time than males performing the same intensity of work. ${ }^{9}$ Most studies have reported that $75 \%$ or more of patients with chronic fatigue syndrome were female. ${ }^{7,10}$ Females suffering diseases, such as rheumatoid arthritis, ${ }^{11}$ poststroke ${ }^{8}$ and acute myocardial infarction, ${ }^{12}$ are significantly more likely to present with fatigue, and their fatigue degree is more serious than that of males. Lower endurance test scores were also found for women than for men in healthy people and in patients, especially in athletes. ${ }^{5,6,13-16}$ These studies are consistent with the fact that female sports records are not as good as those of males, and female work endurance is generally shorter than that of males. These gender differences likely protect females from being overworked and suggest that some hormonal factors, particularly estrogen, are involved in this phenomenon. However, no direct evidence or an underlying mechanism has been clearly established.

Our previous studies reported that the endogenous antifatigue protein orosomucoid (ORM) enhanced muscle endurance. ORM, also known as $\alpha-1$ acid glycoprotein, is an acutephase protein that exhibits a molecular weight of $37-54 \mathrm{kDa}$, a

\footnotetext{
${ }^{1}$ Department of Pharmacology, School of Pharmacy, Second Military Medical University, Shanghai, People's Republic of China and ${ }^{2}$ Suzhou Institute of Systems Medicine, Center for Systems Medicine, Chinese Academy of Medical Sciences, Suzhou, People's Republic of China

${ }^{3}$ Co-first authors.

Correspondence: Professor X Liu or Dr Z-M Luo, Department of Pharmacology, School of Pharmacy, Second Military Medical University, 325 Guohe Road, Yangpu District, Shanghai 200433, People's Republic of China.
}

E-mail: Ixflying@aliyun.com or 285416709@qq.com

Received 28 March 2017; revised 18 September 2017; accepted 11 October 2017 
low pI of $2.8-3.8$ and heavy glycosylation (45\%). The liver primarily synthesizes ORM, but many extrahepatic tissues also produce ORM under various physiological and pathological conditions. ORM exhibits many biological activities, including modulating immunity, binding and carrying drugs, maintaining the capillary barrier and acting as a disease marker. ${ }^{17}$ The role of ORM in cancer, ${ }^{18-20}$ cardiovascular diseases ${ }^{21,22}$ and energy metabolism ${ }^{23}$ was revealed recently. We previously reported that ORM was significantly elevated in the sera, liver and muscle tissues of fatigued rodents (for example, sleepdeprivation, forced swimming and treadmill-running models) and the sera of chronic fatigue syndrome patients. ${ }^{2,24}$ Further studies demonstrated that ORM functioned as an anti-fatigue protein via activation of muscle CCR5 (C-C chemokine receptor type 5) to increase glycogen storage and muscle endurance. ${ }^{2}$ Therefore, we examined whether ORM participated in the differential regulation of fatigue and muscle endurance in males and females.

We found that weaker muscle endurance in females was closely related to reduced ORM induction in response to fatigue. The in vivo and in vitro data demonstrated that estrogen could downregulate ORM expression via estrogen receptors (ERs) and the p38 mitogen-activated protein kinase (MAPK) pathway that decreased muscle endurance. This difference in ORM may be responsible for the gender difference in muscle fatigability.

\section{MATERIALS AND METHODS}

\section{Reagents}

17ק-Estradiol and ICI 182780 (a selective ER degrader) were purchased from Sigma-Aldrich (St Louis, MO, USA). BEZ235 (phosphatidylinositol 3-kinase (PI3K) inhibitor) and SB203580 (MAPK inhibitor) were purchased from Selleck Chemicals (Houston, TX, USA). The antibody against ORM (rat) was purchased from Abcam (Cambridge, UK), and the antibody against ORM (mouse) was obtained from Genway (San Diego, CA, USA). An antibody against glyceraldehyde-3-phosphate dehydrogenase (GAPDH) was purchased from the Beyotime Institute of Biotechnology (Shanghai, China). Secondary antibodies conjugated with IRDye 800CW were purchased from Rockland Immunochemicals (Limerick, PA, USA).

\footnotetext{
Animals

Sprague-Dawley rats weighing $220 \pm 7 \mathrm{~g}$ and C57BL/6 mice weighing $22 \pm 2 \mathrm{~g}$ were purchased from Sino-British SIPPR/BK Laboratory Animals (Shanghai, China). All animals were re-weighed and weight-matched before experiments. ORM1-deficient mice were generated and identified as reported previously. ${ }^{2,23}$ Generally, ORM1 knockout mice were generated through the construction of vectors targeting exons 1 to 5 of the ORM1 gene, transfection and selection of embryonic stem cells (SCR012) and injection of embryonic stem cells into blastocysts of C57BL/6J mice. The resulting chimerical males were bred with female C57BL/6J mice to produce heterozygous mutant mice that were inter-crossed to obtain homozygous and wildtype mice.

All animals were housed under controlled temperature $\left(22 \pm 3^{\circ} \mathrm{C}\right)$ and lighting $(0800-2000 \mathrm{~h})$ conditions with free access to water and a standard rodent diet. Experiments were performed in accordance with the National Institute of Health's 'Guide for the Care and Use of
}

Laboratory Animals' and the approval of the Scientific Investigation Board of the Second Military Medical University.

\section{Cell line culture}

C2C12 cells (mouse muscle myoblasts) were purchased from the Shanghai Institutes for Biological Sciences, Chinese Academy of Sciences (Shanghai, China) and cultured in serum-supplemented media composed of Dulbecco's modified Eagle's medium and 10\% fetal bovine serum. Human Chang liver cells were purchased from BioHermes Biomedical Technology (Wuxi, China) and cultured in RPMI-1640 medium with 10\% fetal bovine serum.

\section{Weight-loaded forced swimming}

Experiments were performed as previously described. ${ }^{2}$ Male and female rats were matched for weight that was controlled at $220 \pm 7$ g. Briefly, mice or rats were placed individually in a water tank ( $46 \mathrm{~cm}$ tall, $20 \mathrm{~cm}$ in diameter, $21-23^{\circ} \mathrm{C}$ ). Experimental animals were forced to swim with a load of $8 \%$ of each body weight attached to the proximal end of the tail. The swimming time was measured from the time when swimming began to the time when animals could not return to the water surface $10 \mathrm{~s}$ after sinking. ${ }^{25}$

\section{Sleep-deprivation fatigue}

Male and female rats were matched for weight that was controlled at $220 \pm 7 \mathrm{~g}$. Rats were deprived of sleep for 5 consecutive days in a cage filled with water to a height of $1.5 \mathrm{~cm}$, as described previously, ${ }^{2,26}$ and the fatigue degree was evaluated using weight-loaded forced swimming.

\section{Treadmill-running}

Male and female rats were matched for weight that was controlled at $220 \pm 7 \mathrm{~g}$. These rats were subjected to running on a treadmill system to exhaustion as described previously. ${ }^{2}$ The rats were forced to run $1 \mathrm{~h}$ per day at a speed of $30 \mathrm{~m} \mathrm{~min}^{-1}$ for 1-week adaptation training. The initial speed was $12 \mathrm{~m} \mathrm{~min}^{-1}$, but it gradually increased every $5 \mathrm{~min}$ at a rate of $3 \mathrm{~m} \mathrm{~min}^{-1}$ until reaching $30 \mathrm{~m} \mathrm{~min}^{-1}$. Rats were forced to run at a speed of $30 \mathrm{~m} \mathrm{~min}^{-1} 1$ week later until the animals were unable to maintain the pace of the treadmill for up to $10 \mathrm{~s}$. The rats were considered exhausted when they could not right themselves when placed in a supine position to check. The exhaustion time was recorded.

\section{Ovariectomy}

Female rats were bilaterally ovariectomized as previously described. ${ }^{27}$ In general, animals were intraperitoneally injected with $30 \mathrm{mg} \mathrm{kg}^{-1}$ of nembutal for anesthesia. The surgical area from the hip to the lowest rib on both sides was shaved and sterilized. A $1.5 \mathrm{~cm}$ incision through the skin, connective tissue and muscle layer was made, and the ovaries were exteriorized with the associated fat pad and fallopian tube. Shamoperated animals (non-ovariectomized) were anesthetized and subjected only to surgery without removal of the ovaries.

\section{Serum biochemical index}

Serum levels of ORM and estradiol were measured using specific enzyme-linked immunosorbent assay (ELISA) kits. The rat ORM ELISA kit was obtained from Abcam. The mouse ORM ELISA kit was obtained from Shanghai Westang Bio-Tech (Shanghai, China). Rat and mouse estradiol (E2) ELISA kits were obtained from Shanghai Westang Bio-Tech Proteins were detected according to the manufacturer's instructions. 


\section{Western blotting for ORM}

Western blotting was performed as previously reported. ${ }^{28}$ Tissues or cells were homogenized on ice and lysed in a lysis buffer that contained a protease inhibitor mixture (Kangchen, Shanghai, China). Protein concentrations were measured using a BCA Protein Assay Kit (Beyotime Institute of Biotechnology). Proteins were separated on sodium dodecyl sulfate-polyacrylamide gels and transferred to a nitrocellulose membrane that was incubated with antibodies specific to ORM. Equal sample loading was confirmed by re-probing the blots for GAPDH.

\section{Fatigue induction in isolated muscle}

Muscle fatigability was measured as previously described. ${ }^{2}$ Mouse and rat soleus muscles were isolated in the integrity without nerve damage. One end of the muscle was fixed, and the other end was linked to a biotic signal collection sensor and processing system (MedLab-U/4CS, MeiYi, Nanjing, China). Evoked contractions of the muscle strips were continuously detected for 3 min under electrical stimulation trains at $10 \mathrm{~V}$ lasting $5 \mathrm{~ms}$ delivered every second for $3 \mathrm{~min}$. The fatigue index was calculated as the ratios of tensions at 1,2 and $3 \mathrm{~min}$ to the initial tension (average of the first 5 contractions).

\section{Reverse transcription-PCR (RT-PCR)}

RT-PCR was performed as previously reported. ${ }^{29}$ Total RNA was extracted from C2C12 cells using Trizol reagent (Invitrogen, Grand Island, NY, USA) and reverse-transcribed using a PrimeScript RT Master Mix Perfect Real-Time Kit (Takara, Otsu, Japan). Real-time quantitative RT-PCR analysis was performed using SYBR RT-PCR kits (Takara). Expression levels were normalized to the control gene GAPDH. The following primer sequences were used: ${ }^{23}$ ORM, 5'-ACACAATAGAGCTTCGGGAGTC-3' (forward), 5'-ATATCTGGC CTTTTGGCATAGA-3' (reverse); and GAPDH, 5' - - GTATGACTCC ACTCACGGCAAA-3' (forward), 5'-GGTCTCGCTCCTGGAAGATG $-3^{\prime}$ (reverse).

\section{Transient transfection and dual luciferase reporter assays}

The ORM luciferase plasmid contained a $-1.4 \mathrm{~kb}$ to $-150 \mathrm{bp}$ fragment of the mouse ORM promoter in front of a luciferase reporter gene $^{30}$ that was kindly provided by Yun Sok Lee (School of Biological Sciences, Seoul National University, Seoul, Korea). HEK293 cells were transfected with the luciferase reporter plasmid and incubated with the indicated doses of estrogen for $24 \mathrm{~h}$. Luciferase activities were measured using the Dual-Luciferase Reporter Assay System (Promega, Madison, WI, USA) according to the manufacturer's instructions. ${ }^{31}$

\section{Statistical analysis}

Student's $t$-test was used to compare two distinct groups. One-way analysis of variance followed by the least significant difference $t$-test was used to compare more than two groups. Interactions were analyzed using two-way analysis of variance followed by the least significant difference $t$ test. Friedman $M$-test was used when the data exhibited heterogeneity of variance. Data are presented as the means \pm s.e.m. $P<0.05$ was considered statistically significant. Experiments were repeated at least three times. Statistical analyses were performed using JMP 4.0.4 software from the SAS Institute (Cary, NC, USA).

\section{RESULTS}

\section{Females exhibited lower muscle endurance than males} in vivo and in vitro

We investigated whether gender difference in fatigue and muscle endurance existed in rodent fatigue models. It is well known that weight-loaded forced-swimming and treadmillrunning models are similar to physical fatigue, and the sleepdeprivation model is generally used in the study of chronic fatigue syndrome patients. ${ }^{32}$ Rats were used to evaluate endurance differences in the three fatigue models because of the high mortality rate in rat sleep-deprivation model. Figures 1a and b show that male rats swam or ran much longer than female rats. Male rats also swam much longer than female rats after 5 days of sleep deprivation (Figure 1c). Gender differences in muscle endurance were also measured in vitro. Slow twitch type I fibers are highly involved in endurance exercise. Therefore, we used isolated soleus muscles. ${ }^{33}$ Repetitive electrical stimulation induces muscle fatigue that manifests as a decline in muscle contraction ability. ${ }^{34}$ The fatigue index measured muscle endurance. This index was calculated as the ratios of tensions at 1,2 or 3 min to the initial tension. Female rats exhibited a significantly lower fatigue index in response to continuous electrical stimulation (Figures 1d and e). These results suggest that females are prone to exhaustion and have lower muscle endurance.
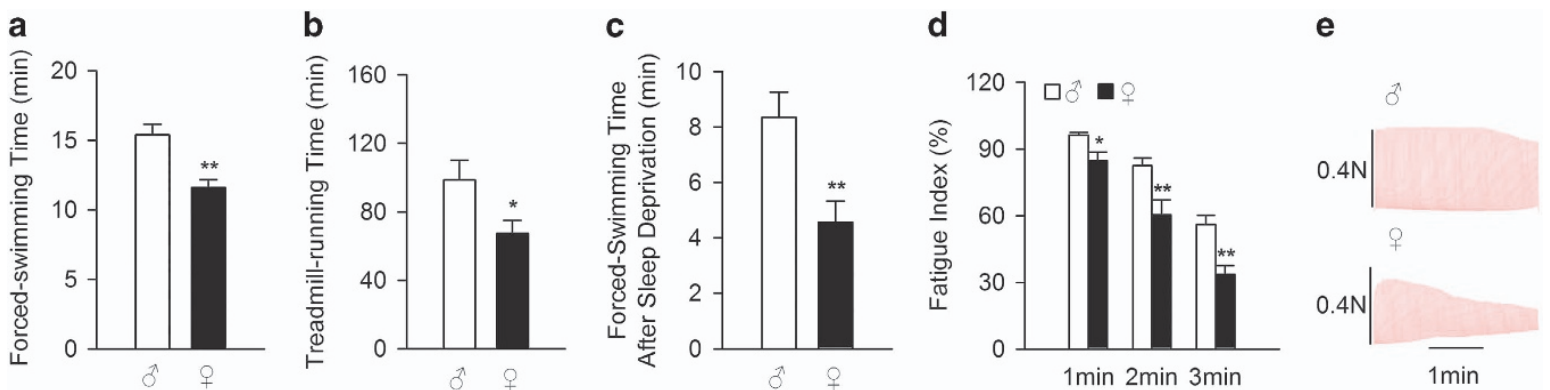

Figure 1 Females exhibit lower muscle endurance than males, as demonstrated by in vivo and in vitro studies. (a) Forced-swimming times of male and female Sprague-Dawley rats ( $n=7-8 /$ group). (b) Treadmill-running times of male and female Sprague-Dawley rats after adaptation trainings for 1 week ( $n=6 /$ group). (c) Forced-swimming times of male and female Sprague-Dawley rats after 5-day sleep deprivation ( $n=5-6 /$ group). (d) Electrically evoked contractions of soleus muscle isolated from male and female Sprague-Dawley rats. The ratio of tension at 1,2 or $3 \mathrm{~min}$ to the initial tension (average of the first 5 contractions) is expressed as the fatigue index. (e) Representative records of the fatigue tests in (d). Male and female rats were matched for weight. Data are presented as the means \pm s.e.m. ${ }^{*} P<0.05$ and ${ }^{*} P<0.01$ by Student's $t$-test. 

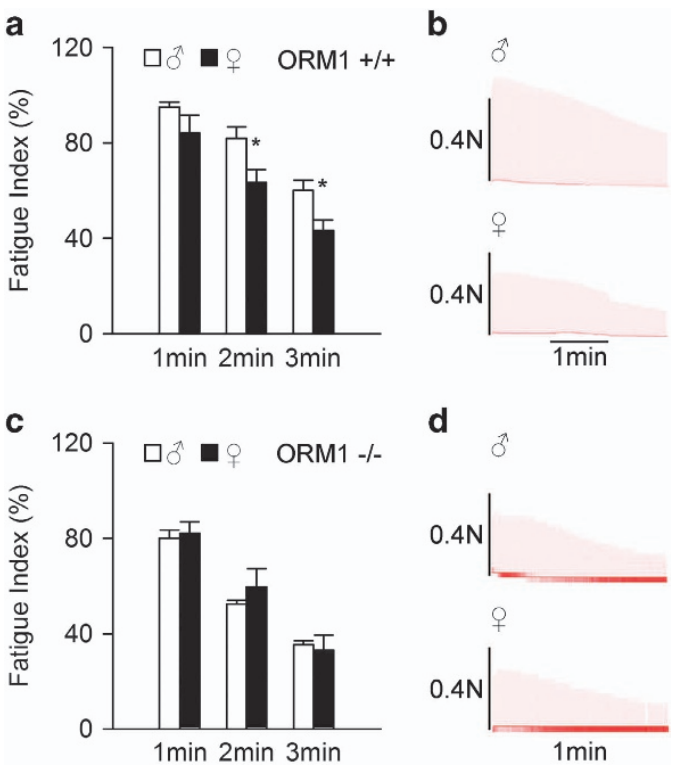

Figure 2 Orosomucoid (ORM) knockout abolished the gender difference in muscle endurance. (a, b) Representative records of electrically evoked contractions of soleus muscle isolated from ORM $1+/+$ mice ( $n=6-7$ per group). (c, d) Representative records of electrically evoked contractions of soleus muscle isolated from ORM1-/ - mice ( $n=5-6$ per group). Data are presented as the means \pm s.e.m. ${ }^{*} P<0.05$ by Student's $t$-test.

\section{ORM knockout abolished the gender difference in muscle endurance}

ORM is an endogenous anti-fatigue protein. ${ }^{2}$ Therefore, we examined the role of ORM in the gender difference in muscle endurance using ORM-deficient mice. There are two isoforms of ORM in humans (ORM1 and ORM2), one isoform in rats (ORM) and three isoforms in mice (ORM1, ORM2 and ORM3). Human and mouse ORM1s are the predominant serum ORM, and it accounts for most of the changes in the ORM pool size during acute-phase responses. ${ }^{17}$ ORM1 knockout mice were constructed as previously reported. ${ }^{2}$ The muscle fatigue index in $\mathrm{ORM}^{+/+}$female mice was lower than that in male mice (Figures $2 \mathrm{a}$ and $\mathrm{b}$ ), similar to the results in rats. However, this difference disappeared in $\mathrm{ORM}^{-/-}$mice (Figures $2 \mathrm{c}$ and $\mathrm{d}$ ), indicating an essential role of ORM in this process.

\section{Fatigue-induced ORM elevation in females was weaker than in males}

We examined whether gender differences in fatigue-induced ORM expression existed. Fatigue was induced as described in Figure 1. Fatigue induced a significant increase of ORM in serum, liver and muscle tissues, and this increase was more obvious in the sleep-deprivation and treadmill-running models. Interestingly, the increase was significantly stronger in male rats than in female rats in response to the same fatigue model (Figures $3 \mathrm{a}$ and $\mathrm{b}$ ), suggesting that the gender difference in fatigue-induced ORM induction participates in the gender difference in muscle endurance. Notably, the induced ORM
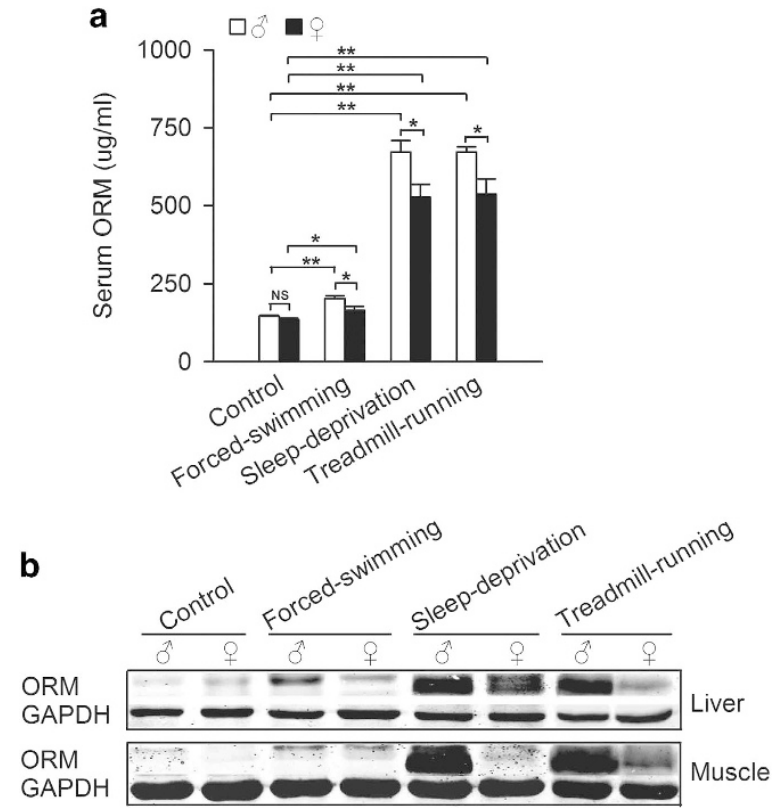

Figure 3 Fatigue-induced orosomucoid (ORM) elevation in females is weaker than males. (a) Serum ORM levels of male and female Sprague-Dawley rats in forced-swimming, sleep-deprivation and treadmill-running fatigue models. (b) Representative western blots of ORM in liver and muscle tissues derived from the rats mentioned in (a) $(n=6-7 /$ group in control group, $n=4$ /group in fatigue models). Data are presented as the means \pm s.e.m. NS, not significant, ${ }^{*} P<0.05$ and $* * P<0.01$ by two-way analysis of variance (ANOVA) with least significant difference (LSD) $t$-test.

levels in serum and tissues were poorly correlated. ORM is synthesized in cells and secreted into the circulation. Tissue ORM expression represents the intracellular level, and serum ORM expression represents the secreted level. The poor correlation likely indicates a nonlinear release of ORM from tissues.

\section{Ovariectomy increased ORM level and muscle endurance}

Hormonal factors, particularly estrogen, may be responsible for the gender difference. Ovariectomy was performed on female Sprague-Dawley rats to examine the possible relationship between endogenous estrogen, ORM expression and muscle endurance. Serum estrogen concentrations decreased significantly 1 month after ovariectomy (Figure 4a), and this decrease was accompanied with elevated ORM level in sera (Figure 4b), liver and muscle (Figure 4c). Swimming time also increased significantly in ovariectomized rats (Figure $4 \mathrm{~d}$ ). Three naturally occurring estrogens in circulation are estrone, 17 $\beta$-estradiol (E2) and estriol, and E2 is the most potent and most commonly used bioactive estrogen in experiments. ${ }^{35}$ Subcutaneous E2 injections $\left(0.5 \mathrm{mg} \mathrm{kg}^{-1}\right)$ for 15 days reversed the above-mentioned effects in ovariectomized rats, indicating a causal relationship between estrogen, ORM and muscle endurance. 
a

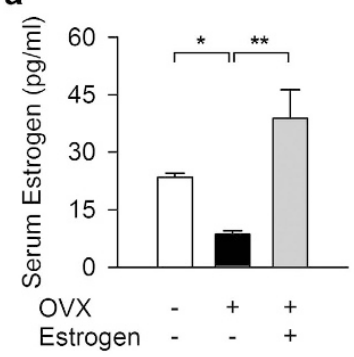

C

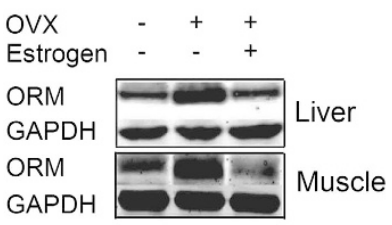

b
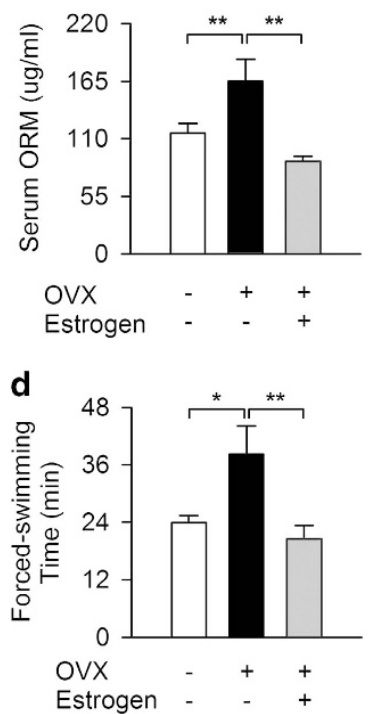

Figure 4 Ovariectomy (OVX) increases orosomucoid (ORM) level and muscle endurance. (a) Serum estrogen level in female SpragueDawley rats with sham operation, OVX without or with E2 replenishment $\left(0.5 \mathrm{mg} \mathrm{kg}^{-1}\right.$ of E2 was subcutaneously injected for 15 days after OVX surgery). (b, c) ORM level in serum (b), liver and muscle (c) of rats treated as mentioned in (a). (d) Forced-swimming times of rats treated as mentioned in (a). $N=8 /$ group. Data are presented as the means \pm s.e.m. ${ }^{*} P<0.05$ and ${ }^{*} * P<0.01$ by oneway analysis of variance (ANOVA) with least significant difference (LSD) $t$-test.

\section{Exogenous estrogen suppressed ORM expression and muscle} endurance

E2 $\left(0.5 \mathrm{mg} \mathrm{kg}^{-1}\right)$ was administered via subcutaneous injection for 15 days (Figure 5a) in wild-type and ORM1 $1^{-1-}$ knockout mice to further investigate the effect of exogenous estrogen on ORM expression and muscle endurance. Figures $5 \mathrm{~b}$ and $\mathrm{c}$ show that estrogen treatment significantly decreased basal ORM levels in the serum, liver and muscle of male and female mice. These mice exhibited significantly decreased swimming times (Figure 5d) and weaker ORM induction in response to swimming-induced fatigue (Figures $5 \mathrm{e}$ and $\mathrm{f}$ ). This long-term estrogen treatment also significantly decreased soleus muscle fatigue index in wild-type mice (Figures $5 \mathrm{~g}$ and $\mathrm{h}$ ) but not in ORM1 knockout mice (Figures 5i and j).

Estrogen inhibited ORM expression in muscle cells and liver cells in vitro

The effect of estrogen on ORM expression was further examined in vitro. E2 treatment decreased ORM protein expression in a dose- and time-dependent manner in $\mathrm{C} 2 \mathrm{C} 12$ muscle cells and Chang liver cells (Figures $6 \mathrm{a}$ and $\mathrm{b}$ ). It also decreased ORM mRNA expression (Supplementary Figures S1a-d). Luciferase reporter results demonstrated that estrogen treatment attenuated mouse ORM promoter activity, indicating a negative effect of estrogen at the ORM transcriptional level (Figure 6c).

Estrogen suppressed ORM expression via an ER-dependent pathway

The effect of estrogen on ORM transcription was investigated further. Most estrogen-mediated signaling pathways are ER dependent, but ER-independent pathways also exist. ${ }^{36}$ ER blockade using the selective ER degrader ICI 182780 completely abolished the inhibitory effect of estrogen on ORM expression in $\mathrm{C} 2 \mathrm{C} 12$ muscle cells and Chang liver cells (Figure 7a and Supplementary Figures S2a and b). ER activation initiates its signal pathways in the nucleus or at the plasma membrane. Nuclear-initiated ER signaling directly regulates target gene transcription via binding to the ER response element, and the membrane-initiated pathway is mediated via the activation of different protein kinases, such as p38 MAPK, $\mathrm{PI} 3 \mathrm{~K}$, extracellular signal-regulated kinase, and phospholipase C. ${ }^{36,37}$ Our results demonstrated that the p38 MAPK inhibitor SB203580 blocked the inhibitory effect of E2 on ORM expression in these cells, and the dual ATP-competitive PI3K and mammalian target of rapamycin inhibitor BEZ235, the extracellular signal-regulated kinase-1/2 inhibitor SCH772984 and the phospholipase C inhibitor U73122 did not block this effect (Figure $7 \mathrm{~b}$ and Supplementary Figures S2c-d and S3). These data suggest that estrogen regulates ORM expression via the ER and its downstream p38 MAPK pathway (Figure 8).

\section{DISCUSSION}

Historical studies of muscle fatigue were performed exclusively in males, and an understanding of potential gender differences in muscle fatigue has been a major focus of investigation only in the past few decades. However, the observed gender differences in muscle fatigue are controversial. Some researchers find no sex-based differences in muscle fatigue, and other studies report that females exhibit greater fatigue resistance than males. ${ }^{38,39}$ More recently, studies demonstrated that men exhibit greater muscle endurance (less fatigability) than women., ${ }^{5,6,13-16}$ The lack of consensus on gender fatigability is likely the result of a variety of factors, including the contraction protocols used to produce fatigue, the index of measurement (that is, endurance time vs decrease in force), age, hormones and other individual indexes of subjects. ${ }^{40}$ Peripheral factors (such as muscle mass, metabolism and perfusion) and central factors (such as force output to motoneurons) for the gender difference in muscle endurance were investigated, but it is not completely understood.

We first verified that females exhibited poorer performance and muscle endurance in different rodent fatigue models in vivo and in vitro. ORM is an endogenous anti-fatigue protein that is induced by fatigue and acts on muscle CCR5 to increase glycogen content and muscle endurance., ${ }^{2,24}$ Therefore, we investigated its role in the gender difference of fatigue and muscle endurance. We found that the poorer performance and muscle endurance in females was accompanied by weaker ORM induction in physical fatigue (forced-swimming and treadmill-running) and mental fatigue (sleep-deprivation) than that in males, suggesting that female hormone regulates ORM. 

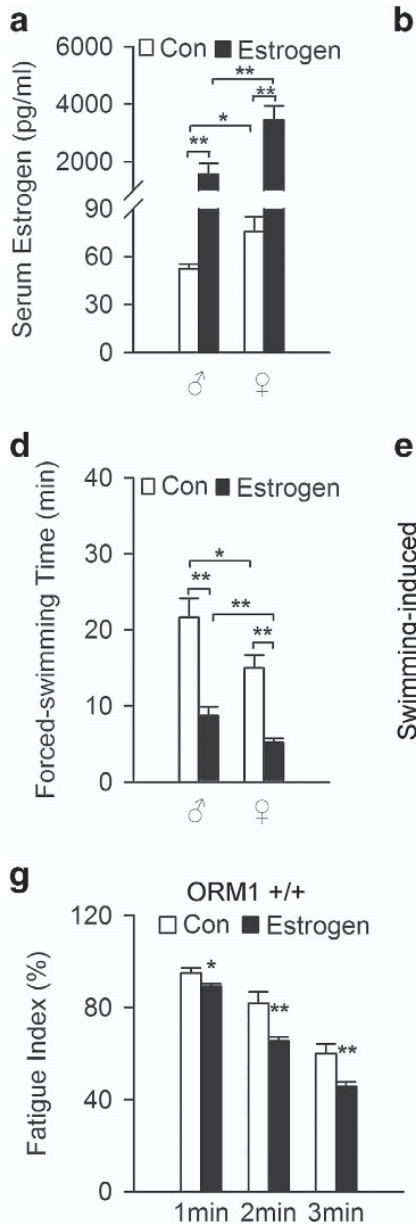

b

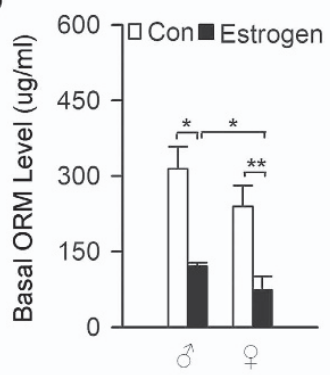

e

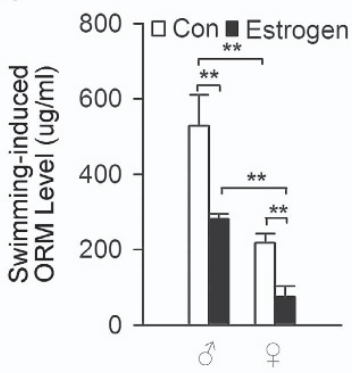

C

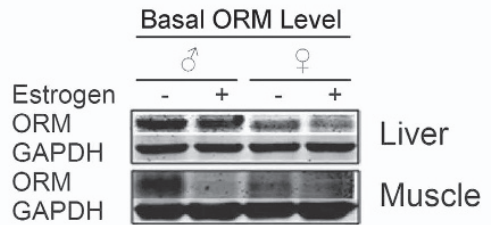

f

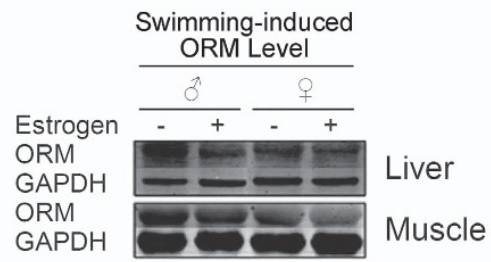

h

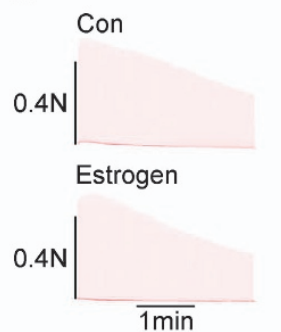

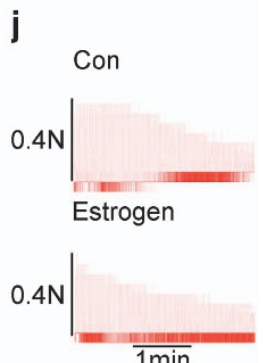

Figure 5 Exogenous estrogen suppresses orosomucoid (ORM) expression and muscle endurance. (a) Estrogen level in the serum of male and female C57BL/6J mice treated with or without subcutaneous injections of E2 for 15 days at a dose of $0.5 \mathrm{mg} \mathrm{kg}-1$ ( $n=7-9 / \mathrm{group}$ ). (b, c) Basal ORM level in serum (b), liver and muscle (c) of mice treated as mentioned in (a) ( $n=3-8 /$ group). (d) Swimming times of mice treated as mentioned in (a) ( $n=10-16 /$ group). (e, f) Swimming-induced ORM levels in serum (e), liver and muscle (f) of mice treated as mentioned in (a) ( $n=4-7 / g r o u p)$. (g, h) Representative records of electrically evoked contractions of soleus muscle isolated from wildtype mice treated as mentioned in (a) ( $n=6-9$ /group). (i, j) Representative records of electrically evoked contractions of soleus muscle isolated from ORM1-deficient mice treated as mentioned in (a) $\left(n=6 /\right.$ group). Data are presented as the means \pm s.e.m. For (e), ${ }^{* *} P<0.01$ by two-way analysis of variance (ANOVA) with least significant difference (LSD) $t$-test. For (a, b, d), ${ }^{*} P<0.05$ and ${ }^{* *} P<0.01$ by Friedman $M$-test.

a

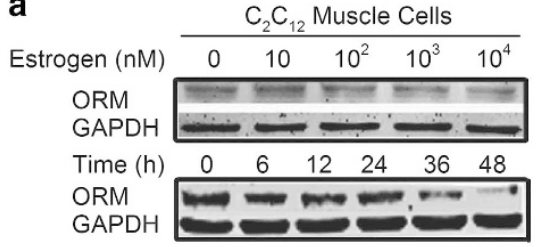

b

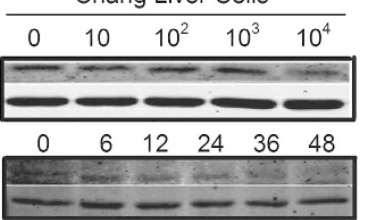

C

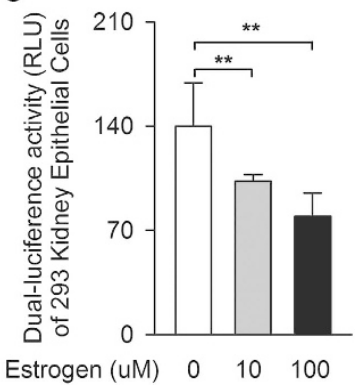

Figure 6 Estrogen inhibits orosomucoid (ORM) expression of muscle cells and liver cells in vitro. (a, b) Representative western blot of ORM in the mouse $\mathrm{C} 2 \mathrm{C} 12$ muscle cell line (a) and human Chang liver cell line (b) treated with the indicated doses of estrogen for $48 \mathrm{~h}$ or $50 \mu \mathrm{m}$ estrogen for the time indicated. (c) Luciferase activity in 293 kidney epithelial cells transfected with the ORM luciferase reporter plasmid and the indicated doses of estrogen for $24 \mathrm{~h}$ ( $n=5$ /group). Western blots are representative of three independent experiments. Data are presented as the means \pm s.e.m. ${ }^{* *} P<0.01$ by one-way analysis of variance (ANOVA) with least significant difference (LSD) $t$-test. 


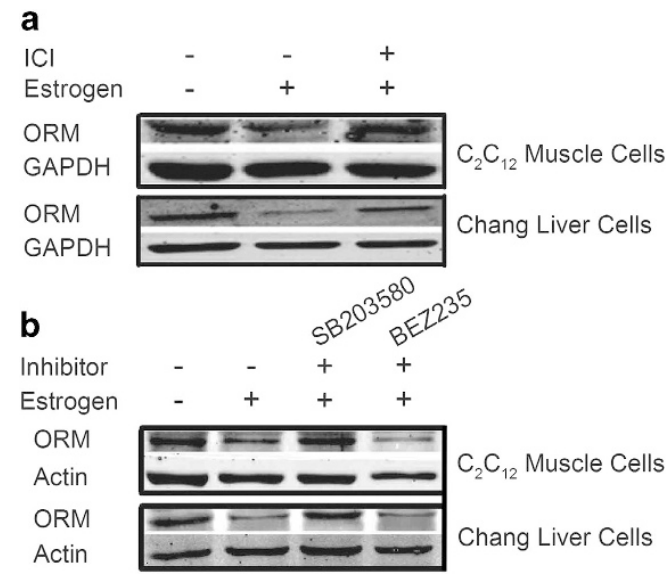

Figure 7 Estrogen suppresses orosomucoid (ORM) expression via the estrogen receptor (ER) and p38 mitogen-activated protein kinase (MAPK) pathway. (a) Representative western blot of ORM in the mouse $\mathrm{C} 2 \mathrm{C} 12$ muscle cell line and human Chang liver cell line treated with vehicle or $50 \mu \mathrm{m}$ estrogen for $48 \mathrm{~h}$ in the presence or absence of $50 \mu \mathrm{M} \mathrm{ICl} 182780$ (a selective ER antagonist). (b) Representative western blot of ORM in the mouse $\mathrm{C} 2 \mathrm{C} 12$ muscle cell line and human Chang liver cell line treated with vehicle or $50 \mu \mathrm{m}$ of estrogen for $48 \mathrm{~h}$ in the presence or absence of $50 \mu \mathrm{M}$ SB203580 (a p38 MAPK inhibitor) or $1 \mu \mathrm{M}$ BEZ235 (a phosphatidylinositol 3-kinase (PI3K) inhibitor). Western blots are representative of three independent experiments.

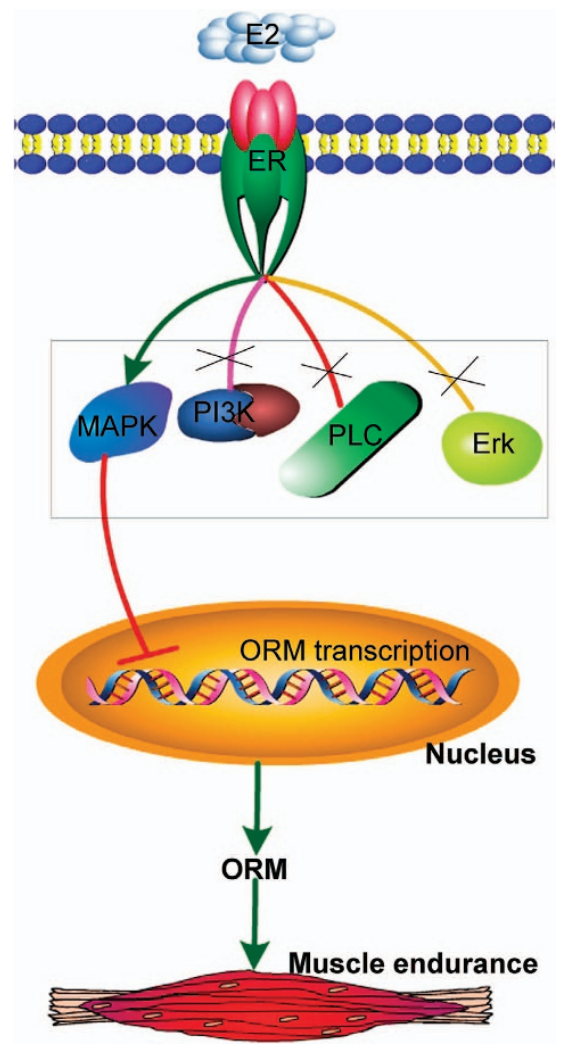

Figure 8 Proposed working model for estrogen regulation of orosomucoid (ORM) in skeletal muscle endurance. Estrogen negatively altered ORM transcription via an estrogen receptoractivated p38 mitogen-activated protein kinase (MAPK) pathway to influence skeletal muscle endurance.
ORM is an acute-phase protein that is upregulated by glucocorticoids, tumor necrosis factor- $\alpha$, interleukin (IL)-1, IL-8, IL-11 and IL-6 cytokines in liver cells from humans, rats, mice and rabbits, and retinoic acids, phenobarbital, farnesoid X receptor, rifampicin and macrolide antibiotics. ${ }^{17}$ The present study found that ORM could be downregulated by estrogen at the transcriptional level via an ER-dependent pathway in liver and muscle tissues. This inhibition of ORM by estrogen may be responsible for the weaker muscle endurance in females. Whether other sex hormones (such as testosterone and progesterone) affect ORM expression is also worthy of future investigation. Notably, basal serum ORM levels were similar between males and females, but the isolated soleus muscle of females exhibited decreased muscle endurance in response to continuous electrostimulation (Figures 1e and 2 ) that might be closely related to the lower basal ORM level in female muscles under physiological conditions (Figure 5c).

The importance of muscle glycogen on muscle performance was demonstrated in a variety of studies, including our previous studies. ${ }^{2,41,42}$ Glycogen depletion is a major factor associated with the onset of fatigue, and most athletes seek methods to increase their muscle glycogen stores in endurance sports. ${ }^{43}$ We found that ORM administration significantly increased muscle glycogen content in mice. ${ }^{2}$ Exercise-trained women exhibit a reduced capacity to store supranormal muscle glycogen levels in response to a diet rich in carbohydrates, and $17 \beta$-estradiol administration to men significantly reduces basal muscle glycogen concentration. ${ }^{44}$ ORM may also mediate the effect of estrogen on muscle glycogen content in human skeletal muscle, but this effect requires further investigation.

The isometric twitch tensions of skeletal muscle are significantly higher in ovariectomized rats than in sham-operated rats or ovariectomized rats that receive estradiol, ${ }^{45}$ indicating a negative role of long-term estrogen in muscle fatigue. Notably, acute $17 \beta$-estradiol treatment of in vitro frog skeletal muscle facilitated the fatigue response to repetitive tetanus stimuli with high frequency, and this direct and quick effect may be related to an increase in the imbalance of $\mathrm{Ca}^{2+}$ turnover in the cytoplasm. ${ }^{46}$ Estrogen also influences glucose uptake via regulation of GLUT4 expression in muscles. ${ }^{47}$ GLUT4deficient mice exhibit a hastened onset of muscle fatigue. ${ }^{48}$ These results indicate a diverse regulation of estrogen in muscle endurance beyond ORM regulation.

Notably, estrogen also regulates ORM glycosylation. Wells et al. ${ }^{49}$ reported that a decrease in the carbohydrate portion of ORM was association with an increase in female sex hormone levels. Brinkman-Van der Linden et al. ${ }^{50}$ reported that oral estrogen treatment increased the degree of branching and decreased fucosylation and sialyl Lewis $\mathrm{X}$ expression compared with control individuals. Changes in the carbohydrate composition of ORM may alter its function because the interaction of ORM and CCR5 is partially dependent on ORM glycosylation. ${ }^{51}$ Whether the estrogen-induced glycosylation changes alters the effect of ORM in muscle endurance should be further examined. 


\section{CONFLICT OF INTEREST}

The authors declare no conflict of interest.

\section{ACKNOWLEDGEMENTS}

We thank Professor Yun Sok Lee (Institute of Molecular Biology and Genetics and School of Biological Sciences, Seoul National University, Seoul, Korea) for providing the mouse ORM promoter luciferase reporter plasmid. We thank Xian-Wei Ma (Department of Immunity, Second Military Medical University) for assistance in performing the ORM promoter reporter assays. We thank Professor Bei Ma (Department of Physiology, Second Military Medical University) for help with fatigue index measurements. This study was supported by grants from the National Natural Science Foundation of China (No. 81273606 , No. 81473259 to XL and No. 81603116 to YS) and the National Science and Technology Major Project (No.

2014ZX09J14103-08C to XL).

Author contributions: XL and DFS designed the experiments. XL supervised the experiments. YS, ZML, ZQ, JJW, PYW, JGY and BHH performed the experiments. YS and ZML created the graphs and wrote the manuscript. XL revised the manuscript. YLY participated in the discussion of the manuscript. All authors analyzed and interpreted experimental data.

\section{PUBLISHER'S NOTE}

Springer Nature remains neutral with regard to jurisdictional claims in published maps and institutional affiliations.

1 Chaudhuri A, Behan PO. Fatigue in neurological disorders. Lancet 2004; 363: 978-988.

2 Lei H, Sun Y, Luo Z, Yourek G, Gui H, Yang Y et al. Fatigue-induced orosomucoid 1 acts on $\mathrm{C}-\mathrm{C}$ chemokine receptor type 5 to enhance muscle endurance. Sci Rep 2016; 6: 18839.

3 Wong R, Lopaschuk G, Zhu G, Walker D, Catellier D, Burton D et al. Skeletal muscle metabolism in the chronic fatigue syndrome. In vivo assessment by $31 \mathrm{P}$ nuclear magnetic resonance spectroscopy. Chest 1992; 102: 1716-1722.

4 Gandevia SC. Spinal and supraspinal factors in human muscle fatigue. Physiol Rev 2001; 81: 1725-1789.

5 Doymaz F, Cavlak U. Relationship between thigh skinfold measurement, hand grip strength, and trunk muscle endurance: differences between the sexes. Adv Ther 2007; 24: 1192-1201.

6 Bianco A, Lupo C, Alesi M, Spina S, Raccuglia M, Thomas E et al. The sit up test to exhaustion as a test for muscular endurance evaluation. Springerplus 2015; 4: 309.

7 Reyes M, Nisenbaum R, Hoaglin DC, Unger ER, Emmons C, Randall B et al. Prevalence and incidence of chronic fatigue syndrome in Wichita, Kansas. Arch Intern Med 2003; 163: 1530-1536.

8 Lerdal A, Bakken LN, Kouwenhoven SE, Pedersen G, Kirkevold M, Finset A et al. Poststroke fatigue-a review. J Pain Symptom Manage 2009; 38: 928-949.

9 Di Milia L, Smolensky MH, Costa G, Howarth HD, Ohayon MM, Philip P. Demographic factors, fatigue, and driving accidents: an examination of the published literature. Accid Anal Prev 2011; 43: 516-532.

10 Prins JB, van der Meer JW, Bleijenberg G. Chronic fatigue syndrome. Lancet 2006; 367: 346-355.

11 Nikolaus S, Bode C, Taal E, van de Laar MA. Fatigue and factors related to fatigue in rheumatoid arthritis: a systematic review. Arthritis Care Res (Hoboken) 2013; 65: 1128-1146.

12 Coventry LL, Finn J, Bremner AP. Sex differences in symptom presentation in acute myocardial infarction: a systematic review and meta-analysis. Heart Lung 2011; 40: 477-491.

13 Hegge AM, Myhre K, Welde B, Holmberg HC, Sandbakk O. Are gender differences in upper-body power generated by elite cross-country skiers augmented by increasing the intensity of exercise? PLOS ONE 2015; 10: e0127509.
14 Ueda HM, Kato M, Saifuddin M, Tabe H, Yamaguchi K, Tanne K. Differences in the fatigue of masticatory and neck muscles between male and female. J Oral Rehabil 2002; 29: 575-582.

15 Constantin-Teodosiu D, Young S, Wellock F, Short AH, Burden RP, Morgan $A G$ et al. Gender and age differences in plasma carnitine, muscle strength, and exercise tolerance in haemodialysis patients. Nephrol Dial Transplant 2002; 17: 1808-1813.

16 Evans K, Refshauge KM, Adams R. Trunk muscle endurance tests: reliability, and gender differences in athletes. J Sci Med Sport 2007; 10: 447-455.

17 Luo Z, Lei H, Sun Y, Liu X, Su DF. Orosomucoid, an acute response protein with multiple modulating activities. J Physiol Biochem 2015; 71: 329-340.

18 Li F, Yu Z, Chen P, Lin G, Li T, Hou L et al. The increased excretion of urinary orosomucoid 1 as a useful biomarker for bladder cancer. Am J Cancer Res 2016; 6: 331-340.

19 Garcia-Munoz A, Bologna-Molina R, A Rodríguez M, Liceaga-Reyes R, Farfan-Morales JE, Aranda-Romo $\mathrm{S}$ et al. Orosomucoid-1 expression in ameloblastoma variants. Int J Mol Cell Med 2016; 5: 49-56.

20 El-Beblawy NM, Andrawes NG, Ismail EA, Enany BE, Abou El-Seoud HS, Erfan MA. Serum and urinary orosomucoid in young patients with type 1 diabetes: a link between inflammation, microvascular complications, and subclinical atherosclerosis. Clin Appl Thromb Hemost 2016; 22: 718-726.

21 Berntsson J, Ostling G, Persson M, Smith JG, Hedblad B, Engstrom G. Orosomucoid carotid plaque, and incidence of stroke. Stroke 2016; 47: 1858-1863.

22 Kaplan P, Vrtovec B, Jug B. Orosomucoid is an independent predictor of prognosis in chronic heart failure. Wien Klin Wochenschr 2016; 128: 870-874.

23 Sun Y, Yang Y, Qin Z, Cai J, Guo X, Tang Y et al. The acute-phase protein orosomucoid regulates food intake and energy homeostasis via leptin receptor signaling pathway. Diabetes 2016; 65: 1630-1641.

24 Sun Y, Zhang ZX, Liu X. Orosomucoid (ORM) as a potential biomarker for the diagnosis of chronic fatigue syndrome (CFS). CNS Neurosci Ther 2016; 22: 251-252.

25 Moriura T, Matsuda H, Kubo M. Pharmacological study on Agkistrodon blomhoffii blomhoffii BOIE. V. anti-fatigue effect of the $50 \%$ ethanol extract in acute weight-loaded forced swimming-treated rats. Biol Pharm Bull 1996; 19: 62-66.

26 Tanaka M, Nakamura F, Mizokawa S, Matsumura A, Nozaki S, Watanabe Y. Establishment and assessment of a rat model of fatigue. Neurosci Lett 2003; 352: 159-162.

27 Ibrahim WW, Safar MM, Khattab MM, Agha AM. 17beta-Estradiol augments antidepressant efficacy of escitalopram in ovariectomized rats: Neuroprotective and serotonin reuptake transporter modulatory effects. Psychoneuroendocrinology 2016; 74: 240-250.

28 Qin Z, Wan JJ, Sun Y, Wu T, Wang PY, Du P et al. Nicotine protects against DSS colitis through regulating microRNA-124 and STAT3. J Mol Med (Berl) 2017; 95: 221-233.

29 Zhai Y, Wu B, Li J, Yao XY, Zhu P, Chen ZN. CD147 promotes IKK/ IkappaB/NF-kappaB pathway to resist TNF-induced apoptosis in rheumatoid arthritis synovial fibroblasts. J Mol Med (Berl) 2016; 94: 71-82.

30 Lee YS, Choi JW, Hwang I, Lee JW, Lee JH, Kim AY et al. Adipocytokine orosomucoid integrates inflammatory and metabolic signals to preserve energy homeostasis by resolving immoderate inflammation. J Biol Chem 2010; 285: 22174-22185.

31 Lee YS, Lee HH, Park J, Yoo EJ, Glackin CA, Choi YI et al. Twist2, a novel $A D D 1 / S R E B P 1 c$ interacting protein, represses the transcriptional activity of ADD1/SREBP1c. Nucleic Acids Res 2003; 31: 7165-7174.

32 Yasui M, Yoshimura T, Takeuchi S, Tokizane K, Tsuda M, Inoue $\mathrm{K}$ et al. A chronic fatigue syndrome model demonstrates mechanical allodynia and muscular hyperalgesia via spinal microglial activation. Glia 2014; 62 : 1407-1417.

33 Vogel J, Figueiredo de Rezende F, Rohrbach S, Zhang M, Schroder K. Nox4 is dispensable for exercise induced muscle fibre switch. PLOS ONE 2015; 10: e0130769.

34 Wust RC, Morse Cl, de Haan A, Rittweger J, Jones DA, Degens H. Skeletal muscle properties and fatigue resistance in relation to smoking history. Eur J Appl Physiol 2008; 104: 103-110.

35 Luo T, Kim JK. The role of estrogen and estrogen receptors on cardiomyocytes: an overview. Can J Cardiol 2016; 32: 1017-1025.

36 Cui J, Shen Y, Li R. Estrogen synthesis and signaling pathways during aging: from periphery to brain. Trends Mol Med 2013; 19: 197-209. 
37 Del Principe D, Ruggieri A, Pietraforte D, Villani A, Vitale C, Straface E et al. The relevance of estrogen/estrogen receptor system on the gender difference in cardiovascular risk. Int J Cardiol 2015; 187: 291-298.

38 Clark BC, Manini TM, The DJ, Doldo NA, Ploutz-Snyder LL. Gender differences in skeletal muscle fatigability are related to contraction type and EMG spectral compression. J Appl Physiol (1985) 2003; 94: 2263-2272.

39 Hicks AL, Kent-Braun J, Ditor DS. Sex differences in human skeletal muscle fatigue. Exerc Sport Sci Rev 2001; 29: 109-112.

40 Enns DL, Tiidus PM. The influence of estrogen on skeletal muscle: sex matters. Sports Med 2010; 40: 41-58.

41 Bertocci LA, Fleckenstein JL, Antonio J. Human muscle fatigue after glycogen depletion: a $31 \mathrm{P}$ magnetic resonance study. J Appl Physiol (1985) 1992; 73: 75-81.

42 Shulman RG, Rothman DL. The "glycogen shunt" in exercising muscle: a role for glycogen in muscle energetics and fatigue. Proc Natl Acad Sci USA 2001; 98: 457-461.

43 Conlee RK. Muscle glycogen and exercise endurance: a twenty-year perspective. Exerc Sport Sci Rev 1987; 15: 1-28.

44 Devries MC, Hamadeh MJ, Graham TE, Tarnopolsky MA. 17beta-estradiol supplementation decreases glucose rate of appearance and disappearance with no effect on glycogen utilization during moderate intensity exercise in men. J Clin Endocrinol Metab 2005; 90: 6218-6225.

45 Suzuki S, Yamamuro T. Long-term effects of estrogen on rat skeletal muscle. Exp Neurol 1985; 87: 291-299.

46 Hatae J. Effects of 17 beta-estradiol on tension responses and fatigue in the skeletal twitch muscle fibers of frog. Jpn J Physiol 2001; 51: 753-759.

47 Faulds MH, Zhao C, Dahlman-Wright K, Gustafsson JA. The diversity of sex steroid action: regulation of metabolism by estrogen signaling. J Endocrinol 2012; 212: 3-12.
48 Gorselink M, Drost MR, de Brouwer KF, Schaart G, van Kranenburg GP, Roemen TH et al. Increased muscle fatigability in GLUT-4-deficient mice. Am J Physiol Endocrinol Metab 2002; 282: E348-E354.

49 Wells C, Bog-Hansen TC, Cooper EH, Glass MR. The use of concanavalin A crossed immuno-affinoelectrophoresis to detect hormone-associated variations in alpha 1-acid glycoprotein. Clin Chim Acta 1981; 109: 59-67.

50 Brinkman-Van der Linden CM, Havenaar EC, Van Ommen CR, Van Kamp GJ, Gooren LJ, Van Dijk W. Oral estrogen treatment induces a decrease in expression of sialyl Lewis $\mathrm{X}$ on alpha 1 -acid glycoprotein in females and male-to-female transsexuals. Glycobiology 1996; 6: 407-412.

51 Atemezem A, Mbemba E, Vassy R, Slimani H, Saffar L, Gattegno L. Human alpha1-acid glycoprotein binds to CCR5 expressed on the plasma membrane of human primary macrophages. Biochem J 2001; 356: 121-128.

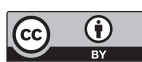

This work is licensed under a Creative Commons Attribution 4.0 International License. The images or other third party material in this article are included in the article's Creative Commons license, unless indicated otherwise in the credit line; if the material is not included under the Creative Commons license, users will need to obtain permission from the license holder to reproduce the material. To view a copy of this license, visit http:// creativecommons.org/licenses/by/4.0/

(C) The Author(s) 2018

Supplementary Information accompanies the paper on Experimental \& Molecular Medicine website (http://www.nature.com/emm) 